\title{
Impact of real-time crowding information: a Stockholm metro pilot study
}

\author{
Yizhou Zhang ${ }^{1} \cdot$ Erik Jenelius $^{1}$ (D) Karl Kottenhoff $^{1}$
}

Accepted: 24 November 2016/Published online: 8 December 2016

(C) The Author(s) 2016. This article is published with open access at Springerlink.com

\begin{abstract}
The paper evaluates the impact of real-time crowding information (RTCI) provision based on a pilot study at a Stockholm metro station. During a 6-day test period, RTCI for each car in the next arriving train was provided through a visual display and speakers. The impact is evaluated in three dimensions: (1) passenger attention is analyzed using video analysis; (2) passenger valuation is evaluated with traveler surveys; (3) passenger action is analyzed with in-vehicle passenger load data. It is estimated that around $25 \%$ of the passengers noticed, understood and considered the provided information useful for their travel decisions. Further, RTCI had a statistically significant positive impact on the boarding distribution between cars and, as a result, on the downstream in-vehicle crowding in the trains. RTCI reduced the share of passengers boarding the first, most crowded car by $4.3 \%$ points for trains that were crowded on arrival, and increased the share of passengers boarding the second, less crowded car by $4.1 \%$ points. The findings also suggest that many passengers may value the provided crowding information positively even though it does not change their travel decisions. The results indicate that RTCI may be a useful technology for public transport operators and agencies for increasing the utilization of available train capacity and reducing crowding.
\end{abstract}

Keywords Crowding $\cdot$ Real-time information · RTCI $\cdot$ Metro $\cdot$ Pilot study

Erik Jenelius

jenelius@kth.se

Yizhou Zhang

yizhouz@kth.se

Karl Kottenhoff

kotten@kth.se

1 Department of Transport Science, KTH Royal Institute of Technology, Teknikringen 10, 10044 Stockholm, Sweden 


\section{Introduction}

As populations increase in many cities world-wide, the ability of public transport systems to provide mobility to the users is put under pressure. Expansion of public transport capacity is often a slow process, and increased travel demand typically leads to increased crowding in vehicles and stations. Generally speaking, crowding refers to the number of travelers in relation to available capacity, although various indicators exist (Li and Hensher 2013). Studies have shown that high crowding may have many negative effects on traveler wellbeing, including stress, anxiety, threat to personal safety and security, and loss of productivity due to lack of seating space (e.g., Beirão and Sarsfield-Cabral 2007; Tirachini et al. 2013). Crowding also affects vehicle dwell times at stations as well as passenger waiting times, which in turn increases variability in headways and reduces reliability. Dwell time impacts of crowding are most significant for buses but exist also for train and metro services (Lin and Wilson 1992; Lam et al. 1999; Qi et al. 2008).

Stated-preference studies show that crowding may significantly increase travelers' value of time savings (Wardman and Whelan 2011; Li and Hensher 2011; Hensher et al. 2011). Recently, revealed-preference studies based on smartcard data have confirmed that travelers consider crowding conditions in their route choices, in addition to travel times and other attributes (Raveau et al. 2014; Kim et al. 2015).

Meanwhile, studies show that passenger loads can be highly unevenly distributed between the cars of trains and metros even during peak hours (TRB 2014). This implies that the effective capacity of the trains is significantly lower than the nominal capacity based on all cars being equally utilized. Kim et al. (2014) investigate the factors that determine whether travelers choose a specific train car intentionally or not in the Seoul metro. In the study, $77 \%$ of the respondents reported choosing a specific car intentionally; among these, $70 \%$ stated that their motivation was to minimize walking distance at the destination station, $17 \%$ sought to minimize walking distance at the origin station, and $13 \%$ stated that they sought to maximize comfort during the trip.

Together, these results suggest that more uniform passenger load distributions between train cars would have positive impacts on passenger wellbeing as well as dwell times, service reliability and on-time performance. Station design, in terms of entrance and exit locations etc., plays an important role for the distribution of crowding. In addition, day-to-day fluctuations in passenger demand will inevitably lead to uneven crowding and non-utilized capacity on some trains. It is therefore valuable to also consider real-time operational strategies, in particular real-time crowding information (RTCI), for equalizing passenger loads between cars.

Real-time information systems for vehicle arrival and travel times are available in many public transport systems world-wide, and have been shown in several studies to have positive effects on perceived waiting times, safety and security, impacts of service disruptions, and general satisfaction (e.g., Dziekan and Kottenhoff 2007; Zhang et al. 2008; Watkins et al. 2011; Cats and Jenelius 2014; Eboli and Mazzulla 2015). Systems providing real-time information about crowding 
in arriving vehicles, however, are less common in practice. In theory, RTCI provision allows travelers to make better informed decisions about whether to board a vehicle or not, and which car of the vehicle to board, based on their preferences for crowding, walking distance, total travel time etc. Thus, RTCI could increase passenger satisfaction and service quality, which would be attractive for public transport authorities and operators. On the other hand, new technology is associated with costs for installation, operations and maintenance, and there may be concerns that it could lead to information overload and confusion for travelers. Thus, there is a strong need for studies that evaluate the effectiveness of RTCI provision for influencing traveler satisfaction, behavior and passenger load distribution. As far as we are aware, however, the impacts of RTCI have not been reported previously in the scientific literature.

This paper investigates the impact of real-time crowding information provision based on a pilot study in the Stockholm metro network. During two weeks in May 2015, an experiment was carried out in which a prototype RTCI system was implemented at Tekniska Högskolan station. RTCI about the next arriving train was communicated to waiting travelers through visual displays and speakers. In this paper, the effectiveness of the system is evaluated with three complementary methods: video analysis of travelers' responses and reactions to the information, surveys among waiting travelers, and passenger load measurements in train cars. The distribution of boarding passengers between cars during the experiment is compared against a control period from the previous year, and the impact of RTCI is statistically evaluated.

The paper is organized as follows. Section 2 describes the Stockholm metro RTCI case study. Section 3 describes the methodology used to evaluate the impact of the RTCI system. Results are given in Sects. 4, and 5 concludes the paper.

\section{RTCI pilot study}

\subsection{Case study setting}

The number of passengers in the Stockholm metro is steadily increasing; in 2014, the annual average daily number of boarding passengers was 1,205,000, an increase of $19 \%$ since 2004 (SL 2015). Several segments of the metro network are subject to significant crowding during the morning and afternoon peaks. The practical capacity (used by the Stockholm public transport authority) of a standard 3-car metro train is 650 passengers, while the technical capacity (obtained from the train manufacturer) is 1200 passengers. On some segments passenger loads during peak hours are consistently close to or even exceeding the practical capacity. However, passenger loads are often unevenly distributed among cars, so that available capacity is not fully utilized even during rush hours. In the afternoon peak, only between $55 \%$ and $90 \%$ of available seats on different metro lines are occupied on average (SL 2015).

Public transport operators in Stockholm are evaluated based on a set of qualityof-service standards defined by the regional public transport authority (SL). Invehicle crowding is one of the quality aspects, where three levels of quality are 
Table 1 Quality-of-service levels of crowding based on utilization of standee areas, used by SL

\begin{tabular}{lll}
\hline Quality of service & Utilization of standee areas, \% & Passenger load per metro car \\
\hline High (green) & $<20$ & $<150$ \\
Average (orange) & $20-40$ & $150-250$ \\
Low (red) & $>40$ & $>250$ \\
\hline
\end{tabular}

Third column shows levels converted to passenger loads in individual metro cars

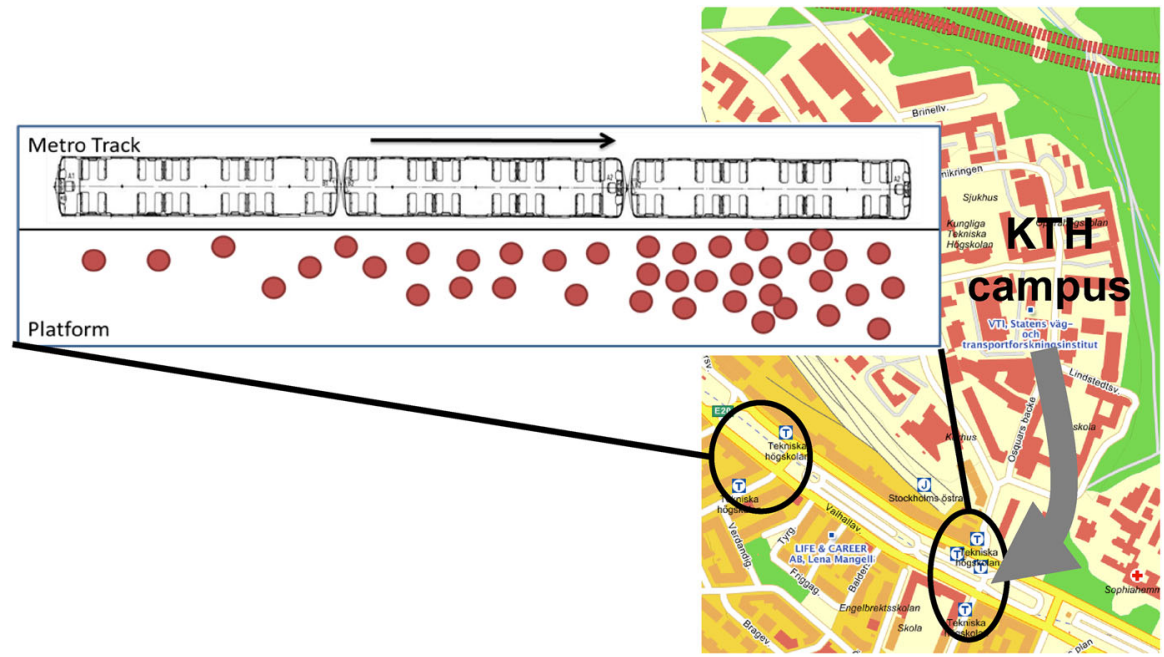

Fig. 1 Layout of Tekniska högskolan metro station. In the afternoon, large passenger flows enter the station from the east entrance near KTH campus, causing a skewed distribution of waiting passengers on the platform

defined based on the utilization of available standee areas as shown in Table 1, second column. These definitions are common for trains, metro and buses. For the purposes of this study, the quality-of-service levels are expressed in terms of the total number of passengers in a standard metro car, based on typical distributions between sitting and standing passengers in the metro (Table 1, third column).

Considering the crowding quality-of-service assessments, there are clear incentives for the metro operator to invest in measures that reduce the inequality of passenger loads between train cars. Towards this goal, a pilot study of the effectiveness of real-time crowding information provision was carried out in May 2015. A screening of potential deployment locations where RTCI provision could be accurate, effective and technically feasible was first conducted (see further Zhang 2015). The platform for southbound trains at Tekniska Högskolan station was selected for the study. The station is located close to the main campus of KTH Royal Institute of Technology and has entrances at both ends of the platform. In the afternoon peak, the distribution of waiting passengers on the platform tends to be heavily skewed with the centre of mass towards the south end (Fig. 1). There are several reasons for the skewness of the distribution. First, during the afternoon peak 

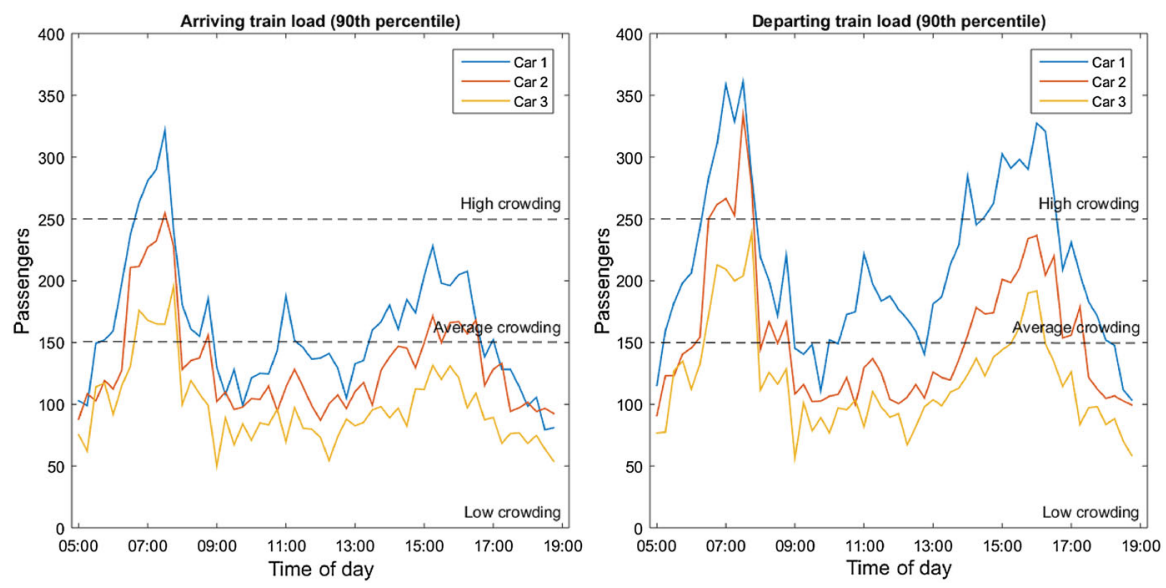

Fig. 2 90th percentile of train passenger load at Tekniska Högskolan metro station across the day for each car separately, based on train car weight measurements from May 2014, Monday-Friday. Left Arriving train load. Right departing train load

hours large passenger flows enter the quite narrow platform from the south entrance near the campus, while few passengers arrive from the north entrance (Fig. 1). Second, several downstream stations, including the central hub T-Centralen, have popular exits located at the south end of the platforms, which means that boarding the first car is attractive for minimizing walking distance and door-to-door travel time.

The station preceding Tekniska Högskolan is Universitetet, which also experiences high travel demand during the afternoon peak. Furthermore, the passenger load distribution in trains arriving to Tekniska Högskolan is already skewed with the highest load typically in car 1 and the lowest load in car 3 (Fig. 2, left). As Fig. 2 illustrates, the crowding quality-of-service often reaches average or low levels during peak hours. Only few passengers alight at Tekniska Högskolan in the afternoon peak. As a result, crowding levels increase significantly and the skewness of the distribution between cars becomes more severe (Fig. 2, right).

\subsection{RTCI provision}

The RTCI pilot study was conducted from Wednesday May 6, 2015 to Wednesday May 13, 2015 (excluding the weekend), each day during afternoon peak hours between 15:30 and 17:30. The collection and provision of RTCI information was managed by an operator located at Tekniska Högskolan station. Real-time crowding information was provided to waiting passengers in two complementing ways:

1. Visual information on overhead screen.

2. Vocal information through speaker system.

Preliminary tests and surveys were performed to find the appropriate design and location of the RTCI display (for further information, see Zhang (2015)). In the 
evaluation it was emphasized that the information should be easily noticed and understood by passengers. The final design and location are shown in Fig. 3. The RTCI was displayed near the south end of the platform where most passengers enter, next to the permanent display of real-time arrival time information. The information was projected onto a screen using an LED projector mounted in the ceiling and connected to a laptop computer. Considering the large share of international travelers from KTH campus, the display included information in both Swedish and English. Each car in the train was shown in color based on the crowding level in the next arriving train. The colors correspond to the three levels of crowding quality-ofservice (Table 1). Thus, green color means low crowding (less than 150 passengers), orange color means average crowding (between 150 and 250 passengers), and red color means high crowding (more than 250 passengers). The arrow indicates the driving direction of the train.

The audio-based information system was employed as a complement to the visual display during parts of each test day. The same information as displayed on the screen was communicated in both Swedish and English spoken language. Depending on the crowding levels of the next train, the message transmitted in English was

1. "Welcome to Tekniska Högskolan. Here is real-time crowding information for the next metro:"

2. "The first train unit is almost full, it is overcrowded/half full, with some standing areas/less crowded, it might have seats left."

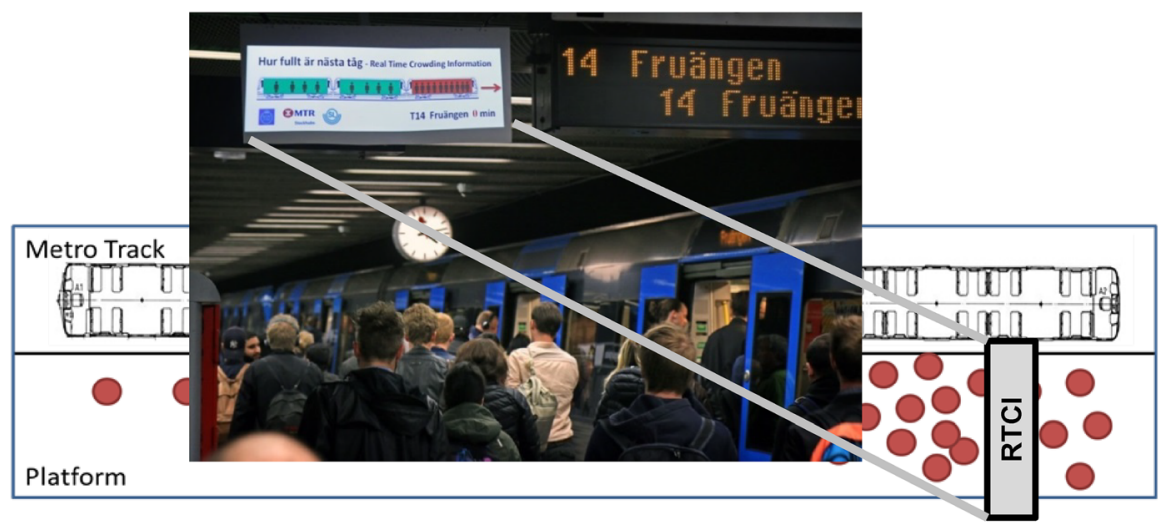

Hur fullt är nästa tåg / Real Time Crowding Information

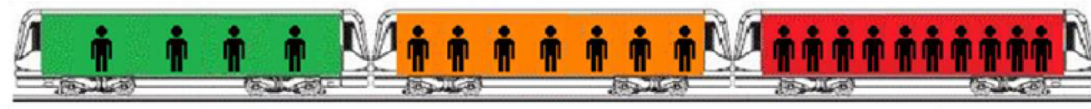

$\mathbf{M T R}$

T14 Fruängen 4 min

Fig. 3 Design and location of the RTCI display 
3. "The second train unit is almost full, it is overcrowded/half full, with some standing areas/less crowded, it might have seats left."

4. "The last train unit is almost full, it is overcrowded/half full, with some standing areas/less crowded, it might have seats left."

5. "We wish you a pleasant journey in Stockholm."

The message in Swedish was similar. The vocal information was transmitted through a floor-mounted speaker installed close to the RTCI screen and controlled from the same laptop computer.

\subsection{RTCI collection}

For the pilot study, automated collection of crowding information was not available in real-time. A simple technical method was used for collecting the crowding information and transmitting it to the operator. An officer stationed at Universitetet station observed the level of crowding quality-of-service in each car (low, average or high according to the standard in Table 1) as the train departed, and reported the information to the operator at Tekniska Högskolan via a handheld transceiver.

The planned headway of the metro line is $5 \mathrm{~min}$, and the normal travel time from Universitetet to Tekniska Högskolan station is $2 \mathrm{~min}$ and $20 \mathrm{~s}$. In order to provide crowding prediction at all times, historical crowding data was combined with the real-time observations. When the next train had not yet departed from the preceding station, the system used historical average crowding levels for the relevant time interval. The historical crowding information was projected on the screen only. When the next train had departed from the preceding station, the information was updated according to the transmitted report, and the speaker (during the periods it was deployed) was also used to spread the information.

The passenger load data used to compute historical crowding levels were obtained from weight measurements in the air suspension system of every train car. The number of passengers in each car was estimated based on an average weight of $78 \mathrm{~kg}$ per passenger including luggage.

\section{Evaluation methodology}

In order to be effective, real-time crowding information should be provided in a way that is noticed and understood by the travelers. In this study, the impact of the RTCI provision during the pilot test is evaluated in three aspects: First, the extent to which passengers noticed the provided information (attention). This is evaluated using video recordings of the passengers waiting on the platform. Second, the extent to which passengers found the provided information comprehensible and useful (valuation). This is evaluated based on surveys conducted with waiting passengers on the platform. Third, the extent to which passengers changed their choice of which train car to board (action). This is evaluated based on passenger load data collected during the test period (collected by USB memory sticks but not available in realtime) and data collected during a reference period the previous year. The evaluation 
Table 2 RTCI impact evaluation methodology

RTCI impact

Evaluation method

Attention: passengers notice information

Video analysis

Valuation: passengers consider information useful

Passenger surveys

Action: passengers change car boarding choice

Train car load data

methodology is summarized in Table 2, and each aspect is described in more detail below.

\subsection{Video analysis}

During three of the six test days (May 7, 8 and 11, 2015), a video camera was used for recording passengers' behavior as they passed below the RTCI screen. If passengers showed any clear reaction such as looking up or pointing to the sign, they are considered to have noticed the sign, otherwise not. The impact of the RTCI provision on passenger attention is evaluated based on the share of passengers noticing the displayed information. The speaker system is evaluated by comparing the share of people noticing the information with the speaker system turned on and off, respectively.

\subsection{Passenger surveys}

To evaluate passengers' attention of the provided RTCI, interviews were conducted with passengers waiting on the platform regarding the projection system and the speaker system. For each system, respondents were asked whether they could understand the conveyed information (yes or no), and to what extent they found the information useful (on a scale from 1 to 5, 1 meaning not useful and 5 meaning very useful). They also had the opportunity to give general comments and feedback about the system. Information about the gender and age of the respondent was also collected.

\subsection{Train car load data}

The extent to which passengers altered their choice of which train car to board is evaluated using passenger load data from the same source as was used to provide historical RTCI information during the test. Information about the passenger load in each car is available for every train departing Universitetet as well as Tekniska Högskolan during the test period. The number of passengers boarding each car at Tekniska Högskolan is not directly observed and needs to be estimated. Considering that very few passengers alight at Tekniska Högskolan during the afternoon peak, the number of passengers $N_{i j}$ boarding car $j=1,2,3$ in train $i$ is estimated by the difference in passenger load after departing from Universitetet, $V_{i j}^{U N T}$, and after departing from Tekniska Högskolan, $V_{i j}^{T E H}$, 


$$
N_{i j}=V_{i j}^{T E H}-V_{i j}^{U N T}, \quad j=1,2,3
$$

The share of passengers $p_{i j}$ boarding each car is thus

$$
p_{i j}=\frac{N_{i j}}{\sum_{j^{\prime}=1}^{3} N_{i j^{\prime}}}, \quad j=1,2,3 .
$$

The impact of RTCI on passengers' choices is assessed by comparing the shares of boarding passengers during the test period with a reference period when the RTCI was not in place. As reference period the same days the previous year are used, i.e., from Wednesday May 7, 2014 to Wednesday May 14, 2014 (excluding the weekend). It is hypothesized that there were no significant changes in passenger behavior between the two periods apart from the introduction of RTCI.

\section{Results}

\subsection{Traveler attention: video analysis}

In total around the behavior of 3000 passengers was analyzed based on the video footage of the platform. The results are summarized in Table 3. When the speaker system was operated, $33.2 \%$ of the passengers noticed the displayed information. When the speaker system was shut down, $27.6 \%$ of the passenger noticed the display. A Chi square test of homogeneity is conducted to test whether the speaker systems had an impact on the share of passengers noticing the display. The test shows that the effect of the speaker system is strongly statistically significant ( $p$ value $<0.001$ ).

Qualitatively, the video analysis further shows that some passengers watching the RTCI screen stood in front of the screen and blocked the path, but overall, the path was wider and passenger flow was smoother while the RTCI system was activated compared with the normal situation.

\subsection{Traveler valuation: Passenger surveys}

In total 118 passengers were asked questions about the projection system. $45 \%$ of the respondents were female and $55 \%$ were male. $47 \%$ were children or young adults (10-25 years old), most of them students in Stockholm, 39\% were 26-45 years old, some traveling with their kids, and 14\% were 46-60 years old. The full survey responses are available in Zhang (2015).

Table 3 Results from the video analysis

\begin{tabular}{llrr}
\hline & Noticed display $(\%)$ & Did not notice display $(\%)$ & Total \\
\hline Speaker system on & $600(33.2)$ & $1204(66.8)$ & 1804 \\
Speaker system off & $363(27.6)$ & $951(72.3)$ & 1314 \\
Total & $963(30.8)$ & $2155(69.2)$ & 3118 \\
\hline
\end{tabular}


Table 4 Interview responses regarding visual RTCI display

\begin{tabular}{lllllll}
\hline Age & 1 (not useful) & 2 (little useful) & 3 (average) & 4 (useful) & 5 (very useful) & Total \\
\hline $10-25$ & 0 & 2 & 7 & 17 & 29 & $55(47 \%)$ \\
$26-45$ & 0 & 1 & 5 & 18 & 22 & $46(39 \%)$ \\
$46-60$ & 0 & 1 & 4 & 10 & 2 & $17(14 \%)$ \\
Total & 0 & $4(3.5 \%)$ & $16(14 \%)$ & $45(39 \%)$ & $53(46 \%)$ & 118 \\
\hline
\end{tabular}

Table 5 Interview responses regarding audio RTCI through speakers

\begin{tabular}{lllllll}
\hline Age & 1 (not useful) & 2 (little useful) & 3 (average) & 4 (useful) & 5 (very useful) & Total \\
\hline $10-25$ & 7 & 17 & 18 & 9 & 4 & $55(49 \%)$ \\
$26-45$ & 7 & 17 & 6 & 10 & 5 & $45(40 \%)$ \\
$46-60$ & 2 & 2 & 2 & 7 & 0 & $13(12 \%)$ \\
Total & $16(14 \%)$ & $4(32 \%)$ & $16(23 \%)$ & $45(23 \%)$ & $53(8.0 \%)$ & 113 \\
\hline
\end{tabular}

According to the interview results, $97 \%$ of all respondents could understand the displayed information on the screen. As shown in Table 4, 46\% thought that the information was very useful for making travel decisions. $4 \%$ of the respondents thought it was little useful and no one thought it was not useful. According to the questionnaire results, most of these respondents were frequent travelers who have short travel distances. To assess whether the perceptions differ among the age groups, a Chi square test of homogeneity is conducted. The test shows that the differences between age groups are not statistically significant ( $\mathrm{p}$ value $=0.138$ ).

113 passengers were asked questions regarding the speaker system. Of the respondents, $45 \%$ were female and $55 \%$ were male, the same distribution as in the survey on the projection system. 49\% were 10-25 years old, 39\% were 26-45 years old, and $12 \%$ were more than 46 years old. Only $8 \%$ of the respondents considered the speaker system to be very useful, while around half (46\%) thought the system was not useful or little useful for them (Table 5). Note, however, that the video analysis showed that the speaker system increased the number of passengers that noticed the visual display.

A Chi square test of homogeneity is conducted to assess whether the perceptions differ among the age groups. The test shows that the differences between age groups are statistically significant at the $10 \%$ significance level but not at the $5 \%$ level ( $\mathrm{p}$ value $=0.0753$ ). It is mainly the oldest age group that differs somewhat from the two other age groups, with larger shares of respondents in both the negative and the positive ends of the scale.

\subsection{Traveler action: train car boarding distribution}

Passenger load data are available for 57 trains during the test period and 72 trains during the reference period of the previous year, in total 129 observations. One additional observation from the reference period is removed from the data set since the estimated number of boarding passengers in one car is negative. The distributions of the passenger load on arriving trains at Tekniska Högskolan are 

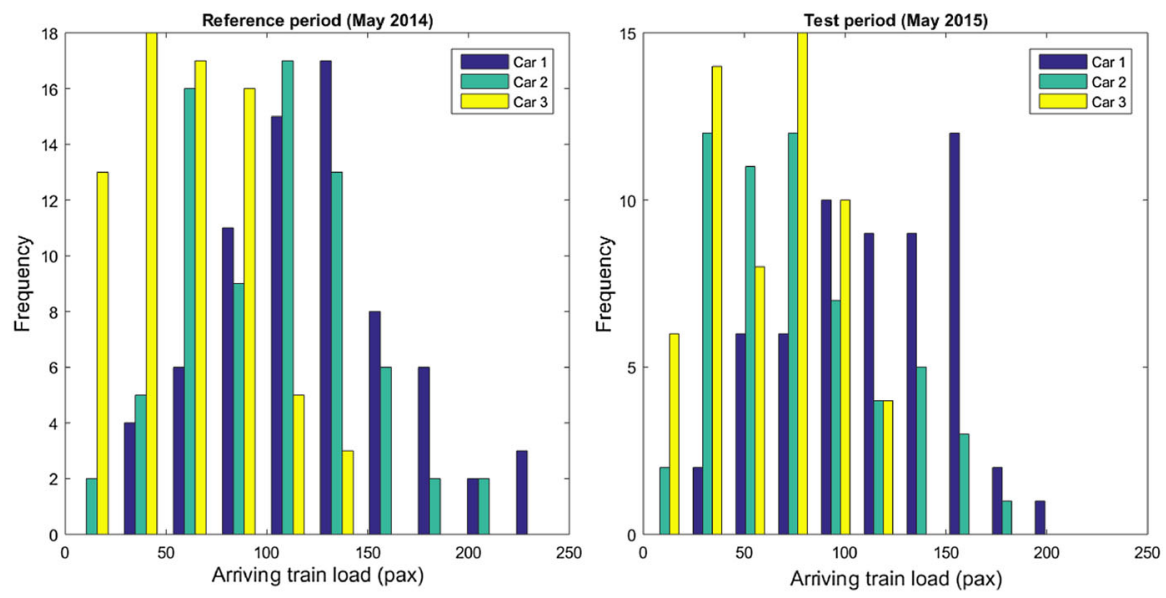

Fig. 4 Distribution of arriving train passenger load at Tekniska Högskolan metro station for each car separately. Left reference period (May 2014). Right RTCI test period (May 2015)

shown in Fig. 4. The distributions are quite similar; during the test period, the average passenger loads in car 1, 2 and 3 were 115, 75.3 and 60.1, respectively. During the reference period, the average passenger loads were 124, 101 and 59.6, respectively. A $t$ test shows that the average share of passengers in car 2 is significantly lower during the test period than the reference period, while the passenger shares in the two other cars are not statistically different at the 5\% level. For trains that were crowded on arrival, meanwhile, there are no statistically significant differences in the shares of passengers between periods. The crowding does not reach the "low" level-of-service, 250 or more passengers, in any arriving train car in the data set.

To evaluate the passenger effect of the RTCI system, the distributions of the share of boarding passengers for each car are shown in Fig. 5. As can be seen, the distributions are similar with the largest share of passengers typically boarding car 1 and the smallest share boarding car 3 in both periods. For car 1, the average boarding share was $55.3 \%$ during the reference period and $54.7 \%$ during the test period. For car 2, the average boarding share increased from $25.4 \%$ during the reference period to $26.5 \%$ during the test period. For car 3, the boarding share changed from 18.8 to $19.2 \%$. The results are summarized in Table 6 .

To test whether the changes in boarding shares are significant, linear regression models

$$
p_{i j}=\beta_{0, j}+\beta_{1, j} \cdot R T C I_{i}+\epsilon_{i j}, \quad i=1, \ldots, 129
$$

are estimated with OLS for each car $j=1,2,3$ separately, where $R T C I_{i}$ is a dummy variable equal to 1 if the observation is from the test period and 0 otherwise. The linear model specification is considered to be suitable as observed boarding share distributions are in the interior of the theoretical lower and upper bounds 0 and $100 \%$, and are approximately distributed Normal (Fig. 5). The results show that the average impact of RTCI across all trains is not statistically significant for any car. 

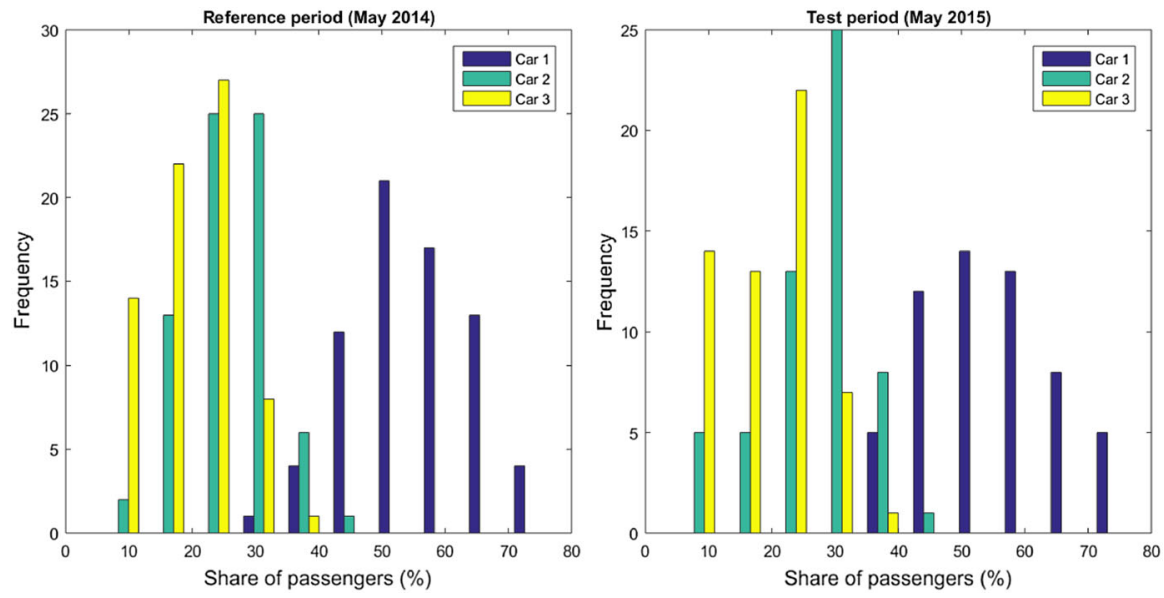

Fig. 5 Distribution of share of boarding passengers at Tekniska Högskolan metro station for each car separately. Left reference period (May 2014). Right RTCI test period (May 2015)

Table 6 Average share of passengers boarding each car during the test period and the reference period

Top: Total across all trains. Middle: Trains with passenger load $<270$. Bottom: Trains with passenger load $\geq 270$

\begin{tabular}{llll}
\hline & Car 1 & Car 2 & Car 3 \\
\hline Total & & & \\
Test period & $54.7 \%$ & $26.5 \%$ & $18.8 \%$ \\
Reference period & $55.3 \%$ & $25.4 \%$ & $19.2 \%$ \\
Difference & $-0.607 \mathrm{pp}$ & $1.036 \mathrm{pp}$ & $-0.429 \mathrm{pp}$ \\
Arriving train uncrowded (load $<270)$ & & \\
RTCI test period & $60.5 \%$ & $22.9 \%$ & $16.6 \%$ \\
Reference period & $57.7 \%$ & $24.7 \%$ & $17.6 \%$ \\
Difference & $2.77 \mathrm{pp}$ & $-1.82 \mathrm{pp}$ & $-0.950 \mathrm{pp}$ \\
Arriving train crowded $(\mathrm{load} \geq 270)$ & & \\
RTCI test period & $48.7 \%$ & $30.2 \%$ & $21.1 \%$ \\
Reference period & $53.0 \%$ & $26.1 \%$ & $20.8 \%$ \\
Difference & $-4.32 \mathrm{pp}$ & $4.05 \mathrm{pp}$ & $0.262 \mathrm{pp}$ \\
\hline
\end{tabular}

A hypothesis is that RTCI provision has a larger impact on passengers' car boarding choices when the arriving train is crowded than when it is not crowded. For crowded trains, RTCI should stimulate passengers to board the less crowded cars. For uncrowded trains, the effect of RTCI should be smaller and may even be in the reversed direction, signaling that choosing a less attractive car is not necessary for avoiding crowding.

Figure 6 shows the share of boarding passengers during the test period and the reference period as functions of the total arriving train passenger load for car 1 and car 2, respectively. For car 1, the boarding share seems to decrease with the arriving train load during both periods; for car 2, the trend is the opposite. To analyze the impact of crowding on boarding shares, an indicator variable $A T C_{i}$ ("arriving train crowded") is introduced, taking value 1 if train $i$ is crowded on arrival and 0 

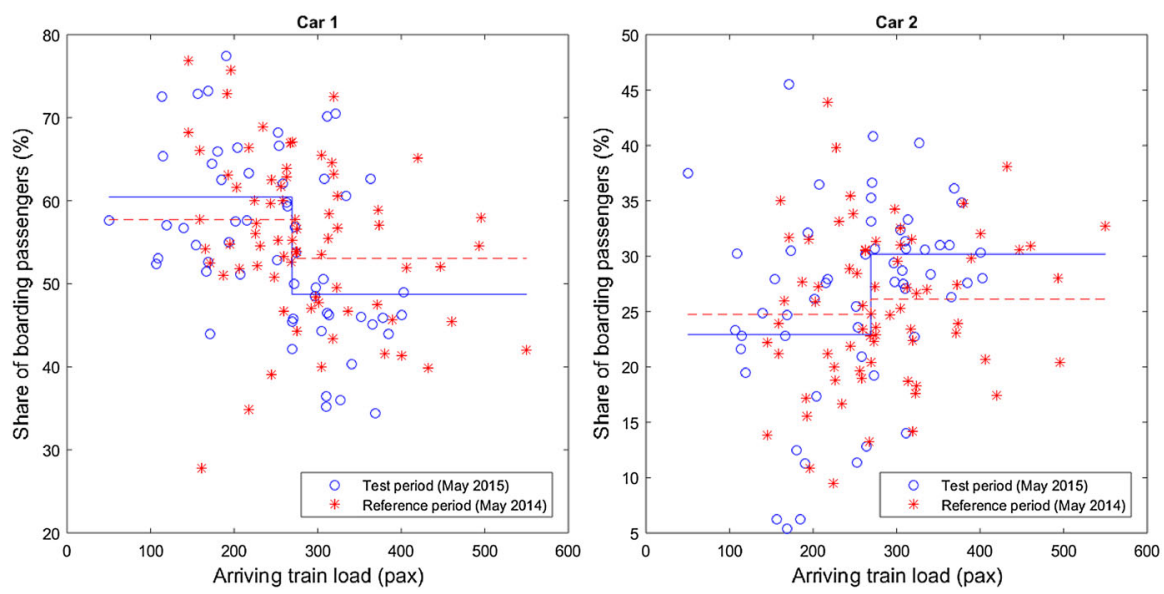

Fig. 6 Share of boarding passengers (per cent) at Tekniska Högskolan metro station as a function of total arriving train passenger load. Left Car 1. Right Car 2. OLS fitted step functions with step at median arriving train load (270 pax)

otherwise. The threshold for crowding is set at the median train passenger load during the test period and the reference period combined, which is 270 passengers. Thus,

$$
A T C_{i}= \begin{cases}1 & \text { if } \sum_{j=1}^{3} V_{i j}^{U N T} \geq 270, \quad i=1, \ldots, 129 . \\ 0 & \text { otherwise }\end{cases}
$$

The average boarding shares in crowded and uncrowded trains, respectively, during the test period and the reference period are shown in Table 6. For car 1, the average boarding share for crowded trains is smaller during the RTCI test period compared to the reference period; for uncrowded trains the boarding share is larger during the test period. The average impact of RTCI is $-4.32 \%$ points for crowded trains and $+2.77 \%$ points for uncrowded trains. Conversely for car 2 , the average boarding share during the test period is smaller for uncrowded trains and larger for crowded trains compared to the reference period. The average impact of RTCI is $4.05 \%$ points for crowded trains and $-1.82 \%$ points for uncrowded trains. Although more complex substitution patterns are also possible, the results indicate that around $4 \%$ of the boarding passengers in crowded situations choose to board the middle car (Car 2) instead of the first car (Car 1) as a result of the RTCI system.

To test the statistical significance of the impact of RTCI and the interaction with crowding, linear regression models

$$
p_{i j}=\beta_{0, j}+\beta_{1, j} \cdot R T C I_{i}+\beta_{2, j} \cdot A T C_{i}+\beta_{3, j} \cdot R T C I_{i} * A T C_{i}+\epsilon_{i j}, i=1, \ldots, 129,
$$

are estimated with OLS separately for each car $j=1,2,3$. The resulting regression lines are plotted in Fig. 6. Table 7 shows the estimation results. For car 1, the estimated impact of RTCI for crowded trains is obtained as $2.77-7.08=-4.32 \%$ 
Table 7 OLS results for the share of passengers boarding car 1 and car 2. 129 observations for both models

\begin{tabular}{|c|c|c|c|c|c|c|}
\hline \multirow[t]{2}{*}{ Parameter } & \multicolumn{3}{|l|}{ Car 1} & \multicolumn{3}{|l|}{ Car 2} \\
\hline & Estimate & $\begin{array}{l}\text { Standard } \\
\text { error }\end{array}$ & $\begin{array}{l}\mathrm{t} \\
\text { statistic }\end{array}$ & Estimate & $\begin{array}{l}\text { Standard } \\
\text { error }\end{array}$ & $\begin{array}{l}\mathrm{t} \\
\text { statistic }\end{array}$ \\
\hline Intercept & $57.7 \%$ & 1.54 & $37.3 * * *$ & $24.7 \%$ & 1.25 & $19.7 * * *$ \\
\hline RTCI $(1=$ yes, $0=$ no $)$ & $2.77 \%$ & 2.30 & 1.20 & $-1.82 \%$ & 1.86 & -0.976 \\
\hline $\begin{array}{l}\text { ATC ( } 1 \text { if load } \geq 270,0 \\
\text { otherwise) }\end{array}$ & $-4.66 \%$ & 2.16 & $-2.16^{* *}$ & $1.39 \%$ & 1.75 & 0.795 \\
\hline RTCI*ATC & $-7.08 \%$ & 3.25 & $-2.18 * *$ & $5.87 \%$ & 2.63 & $2.23 * *$ \\
\hline Num. obs. & 129 & & & 129 & & \\
\hline $\mathrm{R}^{2}$ & 0.184 & & & 0.107 & & \\
\hline
\end{tabular}

$* \mathrm{p}<0.10, * * \mathrm{p}<0.05, * * * \mathrm{p}<0.01$

points. An F test shows that the impact is statistically significant at the $10 \%$ level (p value 0.0620 ). For uncrowded trains, the estimated impact of RTCI is not statistically significant ( $\mathrm{p}$ value 0.231 ).

For car 2, the estimated impact of RTCI for crowded trains is obtained as $-1.82+5.87=4.05 \%$ points. An F-test finds that the impact is statistically significant at the 5\% level (p value 0.0308). For uncrowded trains, the impact of RTCI is not statistically significant ( $p$ value 0.331 ). A corresponding model is estimated also for car 3, where no statistically significant effects of RTCI on the boarding share are found.

The residuals of the linear regression models are analyzed for violations of the OLS assumptions. There are no signs of heteroscedasticity or autocorrelation in the regression model for any of the cars. Furthermore, robust standard errors against heteroscedasticity and autocorrelation are computed, and do not change the statistical significance of the parameter estimates.

To assert the robustness of the results, the regression analysis is repeated using the week preceding the RTCI study as reference period, from Wednesday April 22, 2015 to Wednesday April 29, 2015 (excluding the weekend). The analysis shows that RTCI influences the distributions of boarding passengers in the same directions as compared to the previous year. However, the number of train car load observations during this week is low (in total 28 trains, only few crowded on arrival), and the RTCI impact is not statistically significant.

An alternative model specification is also evaluated, in which the total arriving train passenger load $L_{i}=V_{i, 1}^{U N T}+V_{i, 2}^{U N T}+V_{i, 3}^{U N T}$ is used as independent variable instead of the indicator variable $A T C_{i}$. This specification assumes that the impact of RTCI varies linearly with the arriving train passenger load. Estimation with OLS indicates the same direction of influence from RTCI, but parameters are not statistically significant. The results may suggest the existence of a perceived crowding threshold, close to the median passenger load used here, above which passengers adjust their choice of train car to board in favor of less crowded cars. 


\section{Discussion and conclusion}

The paper evaluated the effects of real-time crowding information provision based on a pilot study in the Stockholm metro. During a 6-day test period, information about the crowding level-of-service in each car of the next arriving train was provided through a visual display and speakers to passengers at Tekniska Högskolan metro station. The impacts were evaluated in three dimensions: (1) passenger attention was analyzed using video analysis; (2) passenger valuation was evaluated with traveler surveys; (3) passenger action was analyzed with in-vehicle passenger load data.

Analysis of video recordings shows that around $31 \%$ of passengers arriving to the platform noticed the information display. The speaker system had a statistically significant positive impact on the share of passengers noticing the display. Based on the passenger surveys, $97 \%$ of the respondents were able to understand the provided information. $85 \%$ of the respondents found the visual information useful or very useful for their travel decisions, while only $31 \%$ found the vocal information useful or very useful. No major differences between different age groups were found. Considering the results together, it can be estimated that around $31 \% \cdot 97 \% \cdot 85 \%=25 \%$ of the passengers noticed, understood and considered the provided information useful for their travel decisions.

Furthermore, analysis of train passenger load data shows statistically significant evidence that RTCI reduced the share of passengers boarding the first, most crowded car by $4.3 \%$ points on trains that were crowded on arrival. Meanwhile, the share of passengers boarding the second, less crowded car increased by $4.1 \%$ points. For trains not crowded on arrival, RTCI worked in the opposite direction and increased the boarding share of car 1 by $2.8 \%$ points and reduced the boarding share of car 2 by $1.8 \%$ points, although these changes are not statistically significant. RTCI did not significantly influence the boarding share of the last, generally least crowded car.

The passenger load analysis shows that RTCI had a measureable, positive impact on the boarding distribution between cars and, as a result, on the downstream invehicle crowding in the trains. The observed change in boarding distribution is smaller than the share of passengers who noticed, understood and considered the RTCI useful according to the video and interview analysis. The findings suggest that many passengers may value the provided crowding information positively even though it does not change their travel decisions. This is in line with previous studies regarding the value of information provision in public transport and passengers' valuations of different trip attributes. For many passengers, minimizing walking distance and door-to-door travel time may be stronger incentives than crowding for choosing a particular train car. Other factors such as on-platform crowding and time constraints may also have limited the extent to which passengers were able to adjust their car boarding choice.

The pilot study suggests that RTCI may be a useful technology for public transport operators and agencies for increasing the utilization of available train capacity and reducing crowding. To generalize the results, further studies should be 
carried out in other locations with varying characteristics of in-vehicle crowding, distribution of waiting passengers on the platform, design of the RTCI system etc. For permanent implementations of RTCI systems, research on technologies for collecting, processing and transmitting crowding information in real-time is an important area. Another direction for further research is short-term prediction of crowding conditions for earlier and possibly more effective RTCI provision.

Acknowledgements The authors would like to thank MTR Nordic and Stockholm County Council Transport Administration for the participation and support of the pilot study and evaluation. The authors also wish to thank two anonymous reviewers for helpful comments on an earlier version of the paper. The evaluation work was financially supported by MTR Nordic and TRENoP Strategic Research Area.

Open Access This article is distributed under the terms of the Creative Commons Attribution 4.0 International License (http://creativecommons.org/licenses/by/4.0/), which permits unrestricted use, distribution, and reproduction in any medium, provided you give appropriate credit to the original author(s) and the source, provide a link to the Creative Commons license, and indicate if changes were made.

\section{References}

Beirão G, Sarsfield-Cabral JA (2007) Understanding attitudes towards public transport and private car: a qualitative study. Transp Policy 14(6):478-489

Cats O, Jenelius E (2014) Dynamic vulnerability analysis of public transport networks: mitigation effects of real-time information. Netw Spat Econ 14(3-4):435-463

Dziekan K, Kottenhoff K (2007) Dynamic at-stop real-time information displays for public transport: effects on customers. Transp Res Part A 41:489-501

Eboli L, Mazzulla G (2015) Relationships between rail passengers' satisfaction and service quality: a framework for identifying key service factors. Public Transp 7:185-201

Hensher DA, Rose JM, Collins AT (2011) Identifying commuter preferences for existing modes and a proposed Metro in Sydney, Australia with special reference to crowding. Public Transp 3:109-147

Kim KM, Hong S-P, Ko S-J, Kim D (2015) Does crowding affect the path choice of metro passengers? Transp Res Part A 77:292-304

Kim H, Kwon S, Wu SK, Sohn K (2014) Why do passengers choose a specific car of a metro train during the morning peak hours? Transp Res Part A 61:249-258

Lam WHK, Cheung C-Y, Lam CF (1999) A study of crowding effects at the Hong Kong light rail transit stations. Transp Res Part A 33:401-415

Li Z, Hensher DA (2011) Crowding and public transport: a review of willingness to pay evidence and its relevance in project appraisal. Transp Policy 18:880-887

Li Z, Hensher DA (2013) Crowding in public transport: a review of objective and subjective measures. J Public Transp 16(2):107-134

Lin T, Wilson NHM (1992) Dwell time relationships for light rail systems. Transp Res Rec 1361:287-295

Qi Z, Baoming H, Dewei L (2008) Modeling and simulation of passenger alighting and boarding movement in Beijing metro stations. Transp Res Part C 16:635-649

Raveau S, Guo Z, Muñoz JC, Wilson NHM (2014) A behavioural comparison or route choice on metro networks: time, transfers, crowding, topology and socio-demographics. Transp Res Part A 66:185-195

SL (2015) Fakta om SL och länet 2014. Stockholm City Council, report SL 2015-1293. In: Swedish. http://www.sll.se/Global/Verksamhet/Kollektivtrafik/Fakta\%20om\%20SL\%20och\%201\%C3\% A4net/Fakta\%20om\%20SL\%20och\%201\%C3\%A4net\%202014.pdf. Accessed 29 Nov 2016

Tirachini A, Hensher DA, Rose JM (2013) Crowding in public transport systems: effects on users, operation and implications for the estimation of demand. Transp Res Part A 53:36-52 
TRB (2014) Transit Capacity and Quality of Service Manual, 3rd edn. Transit Cooperative Highway Research Program (TCRP) Report 165, Transportation Research Board

Wardman M, Whelan GA (2011) Twenty years of rail crowding valuation studies: evidence and lessons from British experience. Transp Rev 31(3):379-398

Watkins KE, Ferris B, Borning A, Rutherford GS, Layton D (2011) Where Is My Bus? Impact of mobile real-time information on the perceived and actual wait time of transit riders. Transp Res Part A 45:839-848

Zhang Y (2015) Real-Time Crowding Information (RTCI) Provision: Impacts and Proposed Technical Solution. M.Sc. Thesis, KTH Royal Institute of Technology, Sweden

Zhang F, Shen Q, Clifton KJ (2008) Examination of traveler responses to real-time information about bus arrivals using panel data. Transp Res Rec 2082:107-115 